\title{
A combinatorial proof for Cayley's identity
}

\author{
Markus Fulmek* \\ Fakultät für Mathematik \\ Oskar-Morgenstern-Platz 1, A-1090 Wien, Austria \\ Markus.Fulmek@Univie.Ac.At
}

Submitted: Jul 15, 2014; Accepted: Nov 14, 2014; Published: Nov 27, 2014

Mathematics Subject Classifications: 05A19

\begin{abstract}
In a recent paper, Caracciolo, Sokal and Sportiello presented, inter alia, an algebraic/combinatorial proof for Cayley's identity. The purpose of the present paper is to give a "purely combinatorial" proof for this identity; i.e., a proof involving only combinatorial arguments. Since these arguments eventually employ a generalization of Laplace's Theorem, we present a "purely combinatorial" proof for this theorem, too.
\end{abstract}

\section{Introduction}

For $n \in \mathbb{N}$, denote by $[n]$ the set $\{1,2, \ldots, n\}$ and let $X=X_{n}=\left(x_{i, j}\right)_{(i, j) \in[n] \times[n]}$ be an $n \times n$ matrix of indeterminates. For $I \subseteq[n]$ and $J \subseteq[n]$, we denote

- the submatrix of $X$ corresponding to the rows $i \in I$ and the columns $j \in J$ by $X_{I, J}$,

- the complementary submatrix of $X_{I, J}$ (which corresponds to the rows $i \in \bar{I}:=[n] \backslash I$ and the columns $j \in \bar{J}:=[n] \backslash J)$ by $X_{\bar{I}, \bar{J}}$.

Let $M=\left\{x_{1} \leqslant x_{2} \leqslant \ldots \leqslant x_{m}\right\}$ be a finite ordered set, and let $S=\left\{x_{i_{1}}, \ldots, x_{i_{k}}\right\} \subseteq M$ be a subset of $M$. We define

$$
\operatorname{sgn}(S \unlhd M):=(-1)^{\sum_{j=1}^{k} i_{j}} .
$$

As pointed out in [2, Section 2.6], the following identity is conventionally but erroneously attributed to Cayley. (Muir [4, vol. 4, p. 479] attributes this identity to Vivanti [6].)

\footnotetext{
*Research supported by the National Research Network "Analytic Combinatorics and Probabilistic Number Theory", funded by the Austrian Science Foundation.
} 
Theorem 1 (Cayley's Identity). Consider $X=\left(x_{i, j}\right)_{(i, j) \in[n] \times[n]}$, and let $\partial=\left(\frac{\partial}{\partial x_{i, j}}\right)$ be the corresponding $n \times n$ matrix of partial derivatives ${ }^{1}$. Let $I, J \subseteq[n]$ with $|I|=|J|=k$. Then we have for $s \in \mathbb{N}$ :

$$
\begin{aligned}
& \operatorname{det}\left(\partial_{I, J}\right)(\operatorname{det}(X))^{s}= \\
& \quad s \cdot(s+1) \cdots(s+k-1) \cdot(\operatorname{det}(X))^{s-1} \cdot \operatorname{sgn}(I \unlhd[n]) \cdot \operatorname{sgn}(J \unlhd[n]) \cdot \operatorname{det}\left(X_{\bar{I}, \bar{J}}\right) .
\end{aligned}
$$

By the alternating property of the determinant, Cayley's Identity is in fact equivalent to the following special case of (1).

Corollary 1 (Vivanti's Theorem). Specialize $I=J=[k]$ for some $k \leqslant n$ in Theorem 1 . Then we have for $s \in \mathbb{N}$ :

$$
\operatorname{det}\left(\partial_{[k],[k]}\right)(\operatorname{det}(X))^{s}=s \cdot(s+1) \cdots(s+k-1) \cdot(\operatorname{det}(X))^{s-1} \cdot \operatorname{det}\left(X_{\overline{[k]},[k]}\right) \text {. }
$$

\section{Combinatorial proof of Vivanti's Theorem}

We may view the determinant of $X$ as the generating function of all permutations $\pi$ in $\mathfrak{S}_{n}$, where the (signed) weight of a permutation $\pi$ is given as $\omega(\pi):=\operatorname{sgn} \pi \cdot \prod_{i=1}^{n} x_{i, \pi(i)}$ :

$$
\operatorname{det}(X)=\sum_{\pi \in \mathfrak{S}_{n}} \omega(\pi)
$$

\subsection{View permutations as perfect matchings}

For our considerations, it is convenient to view a permutation $\pi \in \mathfrak{S}_{n}$ as a perfect matching $m_{\pi}$ of the complete bipartite graph $K_{n, n}$, where the vertices consist of two copies of $[n]$ which are arranged in their natural order; see Figure 1 for an illustration of this simple idea: In the picture, we show the domain of $\pi$ as lower vertices and the image of $\pi$ as upper vertices. It is easy to see that the edges of such perfect matching can be drawn in a way such that all intersections are of precisely two (and not more) edges, and that the number of these intersections equals the number of inversions of $\pi$, whence the sign of $\pi$ is

$$
\operatorname{sgn}(\pi)=(-1)^{\#\left(\text { intersections in } m_{\pi}\right)} .
$$

This simple visualization of permutations and their inversions is already used in $[1, \S 15$, p.32]: We call it the permutation diagram. So assigning weight $x_{i, j}$ to the edge pointing from lower vertex $i$ to upper vertex $j$ and defining the weight $\omega\left(m_{\pi}\right)$ of the permutation diagram $m_{\pi}$ to be the product of the edges belonging to $m_{\pi}$, we may write

$$
\omega(\pi)=(-1)^{\#\left(\text { intersections in } m_{\pi}\right)} \cdot \omega\left(m_{\pi}\right) .
$$

\footnotetext{
${ }^{1} \partial$ is also known as Cayley's $\Omega$-process.
} 


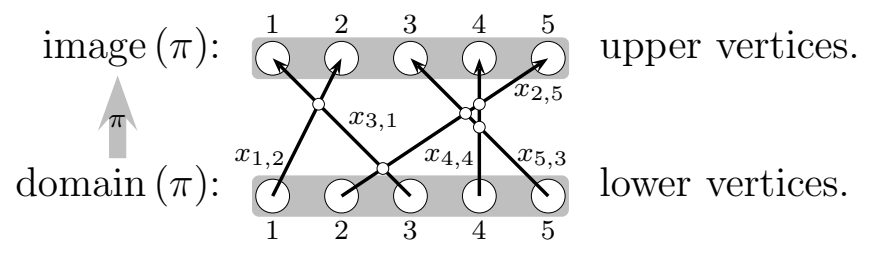

Figure 1: View the permutation $\pi=25143$ as the corresponding perfect matching $m_{\pi}$ in the complete bipartite graph $K_{5,5}$. The intersections of edges are indicated by small circles; they correspond bijectively to $\pi$ 's inversions:

$$
\text { \# (inversions of } \pi)=|\{(1,3),(2,3),(2,4),(2,5),(4,5)\}|=5 .
$$

Assigning weight $x_{i, j}$ to the edge pointing from lower vertex $i$ to upper vertex $j$ gives the contribution of the permutation $\pi$ to the determinant of $X_{5}$ :

$$
\omega(\pi)=(-1)^{5} \cdot x_{1,2} \cdot x_{2,5} \cdot x_{3,1} \cdot x_{4,4} \cdot x_{5,3}
$$

Given this view, the combinatorial interpretation of the $s$-th power of the determinant $\operatorname{det}(X)$ is obvious: It is the generating function of all $s$-tuples $m=\left(m_{\pi_{1}}, \ldots, m_{\pi_{s}}\right)$ of permutation diagrams, where the (signed) weight of such $s$-tuple $m$ is given as

$$
\omega(m)=\prod_{i=1}^{s}(-1)^{\#\left(\text { intersections in } m_{\pi_{i}}\right)} \cdot \omega\left(m_{\pi_{i}}\right) .
$$
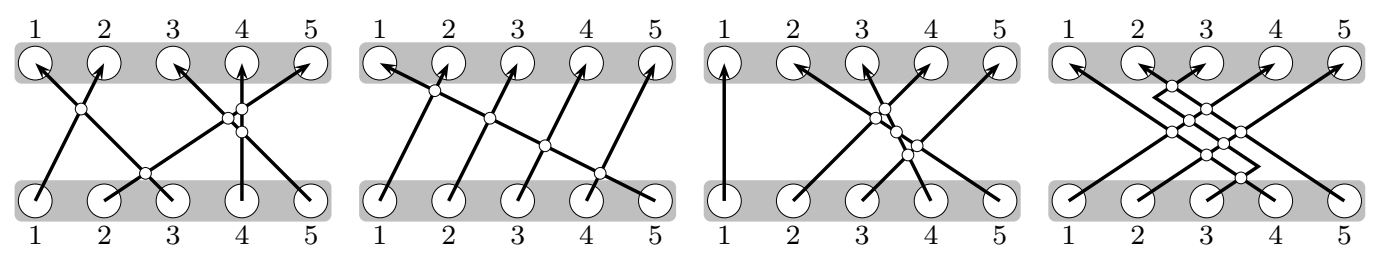

Figure 2: Objects counted by the generating function of a power of a determinant. For $n=5$, the picture shows a typical object of weight

$$
+x_{1,1} x_{1,2}^{2} x_{1,4} x_{2,3} x_{2,4} x_{2,5}^{2} x_{3,1} x_{3,3} x_{3,4} x_{3,5} x_{4,1} x_{4,3} x_{4,4} x_{4,5} x_{5,1} x_{5,2}^{2} x_{5,3},
$$

which is counted by the generating function $\operatorname{det}(X)^{4}$. (The edge connecting lower vertex 3 to upper vertex 3 in the 4 -th (right-most) matching is drawn as zigzag-line, just to avoid intersections of more than two edges in a single point.) 


\subsection{Action of the determinant of partial derivatives}

Next we need to describe combinatorially the action of the determinant $\operatorname{det}\left(\partial_{[k],[k]}\right)$ of partial derivatives. Let $m=\left(m_{\pi_{1}}, \ldots, m_{\pi_{s}}\right)$ be an $s$-tuple of permutation diagrams counted in the generating function $(\operatorname{det}(X))^{s}$, and let $\tau \in \mathfrak{S}_{k}$ : Then the summand

$$
\partial_{\tau}:=\operatorname{sgn}(\tau) \cdot \prod_{i=1}^{k} \frac{\partial}{\partial x_{i, \tau(i)}}
$$

applied to $\omega(m)$ yields

$$
\operatorname{sgn}(\tau) \cdot\left(\prod_{i=1}^{k} \frac{\partial}{\partial x_{i, \tau(i)}}\right) \omega(m)=\operatorname{sgn}(\tau) \cdot c_{\tau, m} \cdot \frac{\omega(m)}{\prod_{i=1}^{k} x_{i, \tau(i)}},
$$

where $c_{\tau, m}$ is the number of ways to choose the set of $k$ edges $\{(i \rightarrow \tau(i)): i \in[k]\}$ from all the edges in $m$ (this number, of course, might be zero). We may visualize the action of $\delta_{\tau}$ as "erasing the edges constituting $\tau$ in $m$ "; see Figure 3 for an illustration.

$$
\tau=(312) \in \mathfrak{S}_{3}:
$$
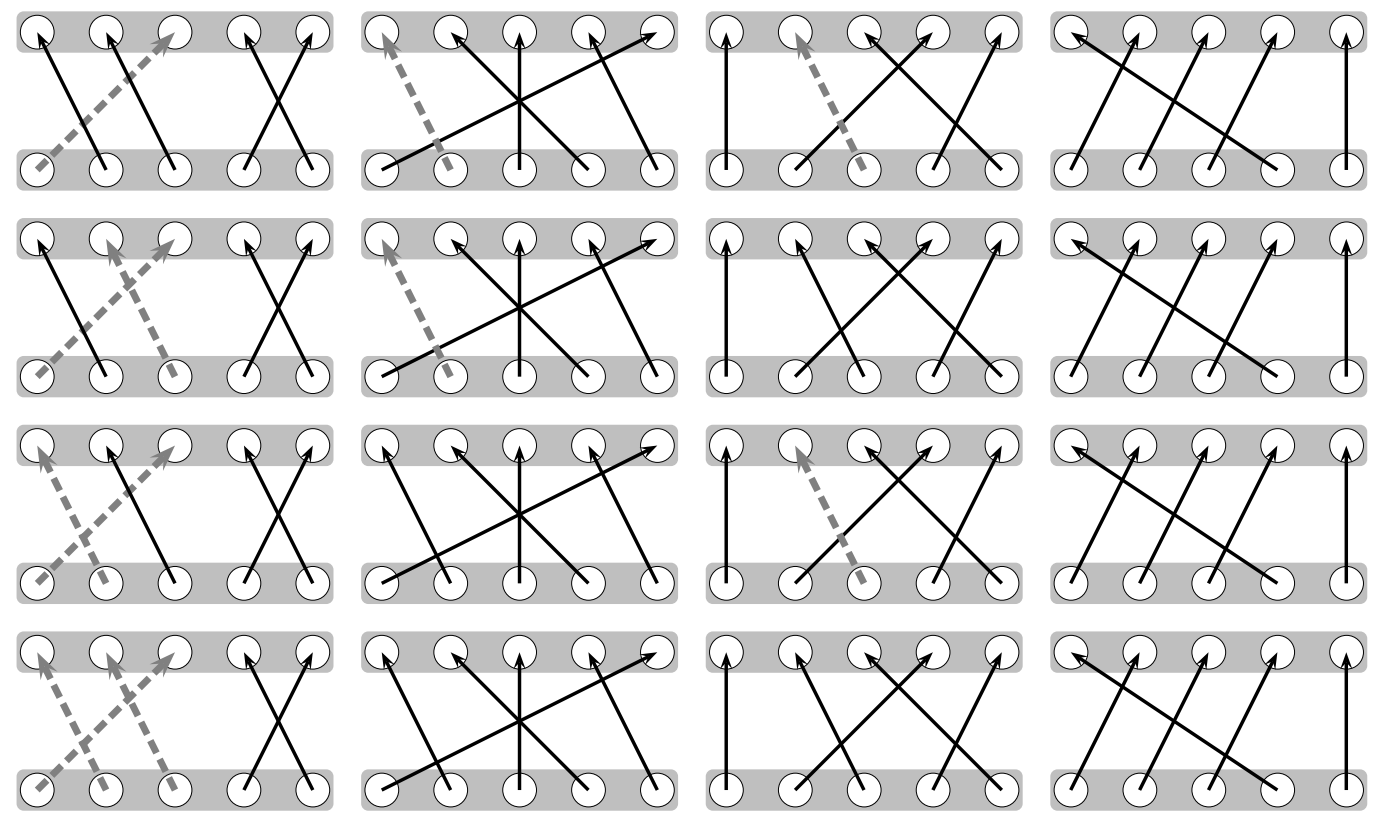

Figure 3: Let $n=5, s=4$ and $k=3$ in Corollary 1. The picture shows four possible ways of "erasing" the edges constituting $\tau \in \mathfrak{S}_{3}$ from the 4-tuple $\left(m_{\pi_{1}}, m_{\pi_{2}}, m_{\pi_{3}}, m_{\pi_{4}}\right)$ of matchings, where $\left(\pi_{1}, \pi_{2}, \pi_{3}, \pi_{4}\right) \in \mathfrak{S}_{5}^{4}$ is $(31254,51324,14253,23415)$. The erased edges are shown as grey dashed lines. 
Hence we have:

$$
\operatorname{det}\left(\partial_{[k],[k]}\right)(\operatorname{det}(X))^{s}=\sum_{m \in \mathfrak{S}_{n}^{s}} \omega(m) \sum_{\tau \in \mathfrak{S}_{k}} c_{\tau, m} \cdot \frac{\operatorname{sgn}(\tau)}{\prod_{i=1}^{k} x_{i, \tau(i)}} .
$$

\subsection{Double counting}

For our purposes, it is convenient to interchange the summation in (3). This application of double counting amounts here to a simple change of view: Instead of counting the ways to choose the set of edges corresponding to $\tau$ from all the edges corresponding to some fixed s-tuple $m$, we fix $\tau$ and consider the set of $m$ 's from which $\tau$ 's edges might be chosen. This will involve two considerations:

- In how many ways can the edges corresponding to $\tau$ be distributed on $s$ copies of the bipartite graph $K_{n, n}$ ?

- For each such distribution, what is the set of compatible $s$-tuples of permutation diagrams?

For example, if $k=3$ and $s=4$ (as in Figure 3), there clearly

- is 1 way to distribute the three edges on a single copy of the 4 bipartite graphs (see the fourth row of pictures in Figure 3), and there are 4 ways to choose such single copy,

- are 3 ways to distribute the three edges on precisely two copies of the 4 bipartite graphs (see the second and third row of pictures in Figure 3), and there are $4 \cdot 3$ ways to choose such pair of copies (whose order is relevant),

- is 1 way to distribute the three edges on precisely three copies of the 4 bipartite graphs (see the first row of pictures in Figure 3), and there are $4 \cdot 3 \cdot 2$ ways to choose such triple of copies (whose order is relevant).

\subsection{Partitioned permutations}

A distribution of the edges corresponding to $\tau \in \mathfrak{S}_{k}$ on $s$ copies of the bipartite graph $K_{n, n}$ may be viewed (see Figure 3)

- as an s-tuple of matchings (some of which may be empty; to stress the fact that these matchings are not perfect, we also call them partial matchings) of $K_{k, k}$

- such that the union of these $s$ partial matchings gives the perfect matching $m_{\tau}$ of $K_{k, k}$.

Clearly, to each such partial matching corresponds a partial permutation $\tau_{i}$, which we may write in two-line notation as follows: 
- the lower line shows the domain of $\tau_{i}$ in its natural order,

- the upper line shows the image of $\tau_{i}$,

- the ordering of the upper line represents the permutation $\tau_{i}$.

We say that each of these $\tau_{i}$ is a partial permutation of $\tau$, and that $\tau$ is a partitioned permutation. We write in short:

$$
\tau=\tau_{1} \star \tau_{2} \star \ldots \star \tau_{s}
$$

For example, the rows of pictures in Figure 3 correspond to the partitioned permutations (written in the aforementioned two-line notation)

- $\left(\begin{array}{l}3 \\ 1\end{array}\right) \star\left(\begin{array}{l}1 \\ 2\end{array}\right) \star\left(\begin{array}{l}2 \\ 3\end{array}\right) \star()$ for the first row,

- $\left(\begin{array}{l}32 \\ 13\end{array}\right) \star\left(\begin{array}{l}1 \\ 2\end{array}\right) \star() \star()$ for the second row,

- $\left(\begin{array}{ll}3 & 1 \\ 1 & 2\end{array}\right) \star() \star\left(\begin{array}{l}2 \\ 3\end{array}\right) \star()$ for the third row,

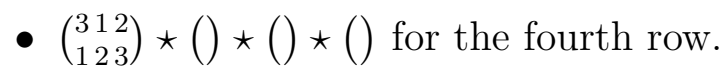

\subsection{Equivalence relation for partitioned permutations}

For any partitioned permutation $\tau=\tau_{1} \star \tau_{2} \star \ldots \star \tau_{s}$, consider the $s$-tuple of the upper rows (in the aforementioned two-line notation) only: We call this $s$-tuple of permutation words the partition scheme of $\tau$ and denote it by $[\tau]$. We say that $\tau=\tau_{1} \star \tau_{2} \star \ldots \star \tau_{s}$ complies to its partition scheme $[\tau]=\left[\tau_{1} \star \tau_{2} \star \ldots \star \tau_{s}\right]$ and denote this by $\tau \subseteq\left[\tau_{1} \star \tau_{2} \star \ldots \star \tau_{s}\right]$.

Now consider the following equivalence relation on the set of partitioned permutations:

$$
\mu=\mu_{1} \star \ldots \star \mu_{s} \sim \nu=\nu_{1} \star \ldots \star \nu_{s}: \Longleftrightarrow[\mu]=[\nu] .
$$

By definition, the corresponding equivalence classes are indexed by a partition scheme, and $\mu=\mu_{1} \star \mu_{2} \star \ldots \star \mu_{s}$ belongs to the equivalence class of $\tau=\tau_{1} \star \tau_{2} \star \ldots \star \tau_{s}$ iff $\mu \subseteq[\tau]$. (For $s>1$, a partitioned permutation $\tau$ is not uniquely determined by $[\tau]$.)

It is elementary to determine the number of these equivalence classes: Think of filling in successively the entries $1,2, \ldots, k$ into the partition scheme $\left[\tau_{1} \star \tau_{2} \star \ldots \star \tau_{s}\right]$. Starting with the empty scheme $[\star \ldots \star \star]$, we find $s$ possibilities to fill in 1 , giving $[\star \ldots \star 1 \star \ldots \star]$. Now there are $s+1$ possibilities to fill in 2, etc.: So the number of these equivalence classes is $s \cdot(s+1) \cdots(s+k-1)$, which is precisely the factor in (2). Our proof will be complete if we manage to show that the generating functions of each of these equivalence classes are the same, namely

$$
(\operatorname{det}(X))^{s-1} \cdot \operatorname{det}\left(X_{\bar{I}, \bar{J}}\right) \text {. }
$$




\subsection{Accounting for the signs}

A necessary first step for this task is to investigate how the sign of a permutation $\pi$ is changed by removing a given partial permutation $\pi_{1}$ : We view this as erasing all the edges belonging to $\pi_{1}$ 's permutation diagram $m_{\pi_{1}}$ from $\pi$ 's permutation diagram $m_{\pi}$; see again Figure 3.

Lemma 1. Let $\pi \in \mathfrak{S}_{n}$ be a partitioned permutation $\pi=\pi_{1} \star \pi_{2}$, where $\pi_{1}$ is the partial permutation

$$
\pi_{1}=\left(\begin{array}{cccc}
\pi\left(i_{1}\right) & \pi\left(i_{2}\right) & \cdots & \pi\left(i_{k}\right) \\
i_{1} & i_{2} & \cdots & i_{k}
\end{array}\right)
$$

(with $\left\{i_{1} \leqslant i_{2} \leqslant \cdots \leqslant i_{k}\right\} \subseteq[n]$ ). Clearly, $\pi_{2}$ is the permutation corresponding to the matching $m_{\pi}$ with edges $\left(i_{1}, \pi\left(i_{1}\right)\right),\left(i_{2}, \pi\left(i_{2}\right)\right), \ldots,\left(i_{k}, \pi\left(i_{k}\right)\right)$ erased, which we also denote by $\pi \backslash \pi_{1}$. Then we have

$$
\operatorname{sgn}(\pi)=(-1)^{\sum_{j=1}^{k} \pi\left(i_{k}\right)-i_{k}} \cdot \operatorname{sgn}\left(\pi_{1}\right) \cdot \operatorname{sgn}\left(\pi_{2}\right) .
$$

If we denote $I=\left\{i_{1}, \ldots, i_{k}\right\}$ and $J=\left\{\pi\left(i_{1}\right), \ldots, \pi\left(i_{k}\right)\right\}$, we may rewrite this as

$$
\operatorname{sgn}(\pi)=\operatorname{sgn}(I \unlhd[n]) \cdot \operatorname{sgn}(J \unlhd[n]) \cdot \operatorname{sgn}\left(\pi_{1}\right) \cdot \operatorname{sgn}\left(\pi \backslash \pi_{1}\right) .
$$

Proof. View the permutation diagram $m_{\pi}$ of $\pi=\pi_{1} \star \pi_{2}$ as a bicoloured perfect matching of $K_{n, n}$, where the edges and vertices corresponding to $\pi_{1}$ are coloured green and the edges and vertices corresponding to $\pi_{2}$ are coloured red (see Figure 4). Clearly,

- the set $I$ is the set of (the labels of the) lower green vertices,

- the set $J$ is the set of (the labels of the) upper green vertices.

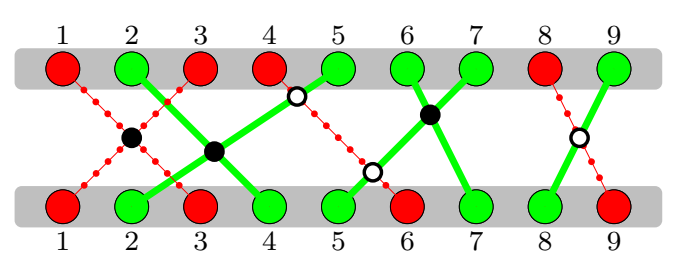

Figure 4: The bicoloured permutation diagram $m_{\pi}$ corresponding to the partitioned permutation $\pi=\pi_{1} \star \pi_{2}$, where

$$
\pi_{1}=\left(\begin{array}{l}
52769 \\
24578
\end{array}\right) \text { and } \pi_{2}=\left(\begin{array}{l}
3148 \\
1369
\end{array}\right)
$$

The edges corresponding to $\pi_{1}$ are shown as green (solid) lines, the edges corresponding to $\pi_{2}$ are shown as red (dotted) lines. The inactive intersections (of green/red edges) are indicated by small white circles, the other intersections (of green/green or red/red edges) are indicated by small black circles.

Note that the intersections in $m_{\pi}$ come in three flavours: 
- intersections of two green edges (which are accounted for in $\operatorname{sgn}\left(\pi_{1}\right)$ ),

- intersections of two red edges (which are accounted for in $\operatorname{sgn}\left(\pi_{2}\right)$ ),

- intersections of a green and a red edge: Since they do not contribute to the signs, let us call them the inactive intersections.

We will prove (4) by showing the following two statements:

1. The parity of the number of inactive intersections depends only on the sets $I$ and $J$, i.e., on the positions of the (lower and upper) green vertices.

2. The number of inactive intersections equals $\sum_{j=1}^{k}\left|\pi\left(i_{j}\right)-i_{j}\right|$ (which, of course, is equal to $\sum_{j=1}^{k} \pi\left(i_{j}\right)+\sum_{j=1}^{k} i_{j}$ modulo 2$)$ in the case that $\pi_{1}: I \rightarrow J$ and $\pi_{2}$ : $([n] \backslash I) \rightarrow([n] \backslash J)$ are the unique order-preserving bijections (i.e, there are only inactive intersections; see Figure 6).

For the first statement, consider two edges $e_{1}$ and $e_{2}$ of the same colour, where $e_{1}=(a, d)$ and $e_{2}=(b, c)$ connect lower vertices $a$ and $b$ with upper vertices $d$ and $c$, respectively, and look at the effect of replacing these edges by $e_{1}^{\prime}=(a, c)$ and $e_{2}^{\prime}=(b, d)$ : It is easy to see that an edge $e$ of the other colour

- has an even number of intersections with $e_{1}$ and $e_{2}$ (i.e., intersects neither of them or both of them) if and only if it has an even number of intersections with $e_{1}^{\prime}$ and $e_{2}^{\prime}$

- has an odd number of intersections with $e_{1}$ and $e_{2}$ (i.e., intersects exactly one of them) if and only if it has an odd number of intersections with $e_{1}^{\prime}$ and $e_{2}^{\prime}$.

See Figure 5 for an illustration: Note that replacing edges $e_{1}, e_{2}$ by $e_{1}^{\prime}, e_{2}^{\prime}$ corresponds to multiplying $\pi_{1}$ with the transposition $(c, d)$, and by multiplying with a sequence of appropriate transpositions, we can remove all inversions from $\pi_{1}$ and $\pi_{2}$; and this operation does not change the parity of the number of inactive intersections.
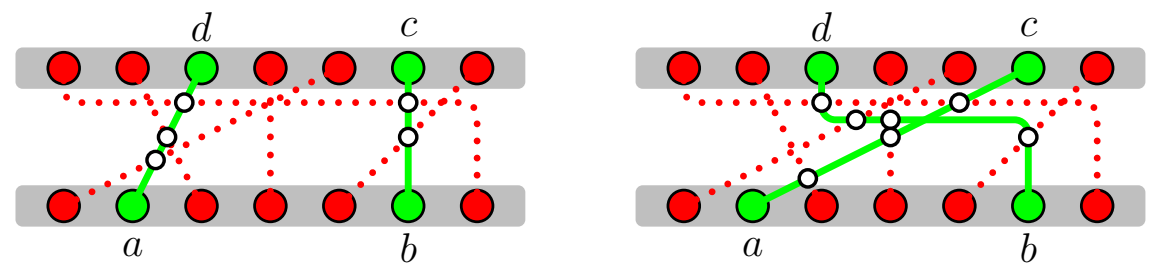

Figure 5: The left picture shows the green edges (shown as solid lines) $e_{1}=(a, d)$ and $e_{2}=(b, c)$, which are replaced by the green edges $e_{1}^{\prime}=(a, c)$ and $e_{2}^{\prime}=(b, d)$ in the right picture: Observe that for every red edge $e$ (shown as dotted line) the parities of the numbers of intersections with $\left\{e_{1}, e_{2}\right\}$ and $\left\{e_{1}^{\prime}, e_{2}^{\prime}\right\}$ are the same. (Some edges are drawn as curved lines here for graphical reasons.) 
For the second statement, simply have a look at Figure 6 and observe that in the case where neither $\pi_{1}$ nor $\pi_{2}$ have inversions, $\left|\pi\left(i_{j}\right)-i_{j}\right|$ is the number of intersections of the $j$-the green edge with red edges.

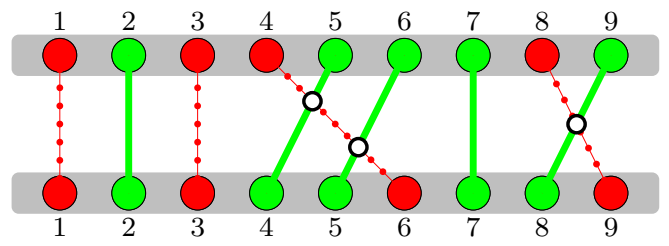

Figure 6: Partitioned permutation $\pi^{\prime}=\pi_{1}^{\prime} \star \pi_{2}^{\prime}$, where both $\pi_{1}^{\prime}$ and $\pi_{2}^{\prime}$ have no inversions; i.e., there are only inactive intersections in the bicoloured permutation diagram $m_{\pi_{1}^{\prime} \star \pi_{2}^{\prime}}$.

\subsection{Sums of (signed) products of minors}

Now consider a fixed equivalence class in the sense of section 2.5, which is indexed by a partition-scheme

$$
\left[\tau_{1} \star \tau_{2} \star \cdots \star \tau_{s}\right] .
$$

We want to compute the generating function $G_{[\tau]}$ of this equivalence class: Clearly, we may concentrate on the nonempty partial permutations; so w.l.o.g. we have to consider the partition-scheme

$$
\left[\tau_{1} \star \tau_{2} \star \cdots \star \tau_{m}\right]
$$

which consists only of nonempty partial permutations $\tau_{j}$ for $1 \leqslant j \leqslant m \leqslant s$. For any $\sigma \in \mathfrak{S}_{k}$ with $\sigma \subseteq\left[\tau_{1} \star \tau_{2} \star \cdots \star \tau_{m}\right]$, such partition scheme corresponds to a unique ordered partition of the image of $\sigma$ :

image $(\sigma)=[k]=\left(\operatorname{image}\left(\tau_{1}\right)\right) \dot{U}\left(\operatorname{image}\left(\tau_{2}\right)\right) \dot{\cup} \cdots \dot{U}\left(\right.$ image $\left.\left(\tau_{m}\right)\right)=J_{1} \dot{\cup} J_{2} \dot{\cup} \cdots \dot{U} J_{m}$, and any specification of a compatible ordered partition $\mathbf{I}_{[J]}=\left(I_{1}, I_{2}, \ldots, I_{m}\right)$, i.e.,

$$
[k]=I_{1} \dot{\cup} I_{2} \dot{\cup} \ldots \dot{\cup} I_{m} \text { where }\left|I_{l}\right|=\left|J_{l}\right|, l=1, \ldots, m,
$$

uniquely determines such $\sigma$, which we denote by $\sigma\left(\mathbf{I}_{[J]},[\tau]\right)$.

Equation (4) gives the sign-change caused by erasing the edges corresponding to $\tau_{l}$ (with respect to any permutation in $\mathfrak{S}_{n}$ which contains $\tau_{l}$ as a partial permutation), whence we can write the generating function as

$$
\begin{aligned}
G_{[\tau]}= & \operatorname{det}(X)^{s-m} \\
& \times \sum_{\mathbf{I}_{[J]}} \operatorname{sgn}\left(\sigma\left(\mathbf{I}_{[J]},[\tau]\right)\right) \cdot \prod_{l=1}^{m}\left(\operatorname{sgn}\left(\tau_{l}\right) \cdot \operatorname{sgn}\left(I_{l} \unlhd[n]\right) \cdot \operatorname{sgn}\left(J_{l} \unlhd[n]\right) \cdot \operatorname{det}\left(X_{\bar{I}_{l}, \bar{J}_{l}}\right)\right),
\end{aligned}
$$


where the sum is over all compatible partitions $\mathbf{I}_{[J]}$. (The factor $\operatorname{sgn}\left(\sigma\left(\mathbf{I}_{[J]},[\tau]\right)\right)$ comes from the determinant of partial derivatives.) Clearly,

$$
\left(\prod_{l=1}^{m} \operatorname{sgn}\left(I_{l} \unlhd[n]\right)\right) \cdot\left(\prod_{l=1}^{m} \operatorname{sgn}\left(J_{l} \unlhd[n]\right)\right)=1,
$$

so it remains to show that

$$
\sum_{\mathbf{I}_{[J]}} \operatorname{sgn} \sigma\left(\mathbf{I}_{[J]},[\tau]\right) \cdot \prod_{l=1}^{m}\left(\operatorname{sgn}\left(\tau_{l}\right) \cdot \operatorname{det}\left(X_{\bar{I}_{l}, \overline{J_{l}}}\right)\right)=\operatorname{det}(X)^{m-1} \operatorname{det}\left(X_{\overline{[k]}, \overline{[k]}}\right) .
$$

This, of course, is true for $m=1$. We proceed by induction on $m$.

For any ordered partition $S_{1} \dot{\cup} S_{2} \dot{\cup} \ldots \dot{\cup} S_{m}=[k]$, we introduce the shorthand notation

$$
\mathbf{S}_{l}:=[k] \backslash\left(S_{1} \dot{\cup} S_{2} \dot{\cup} \ldots \dot{U} S_{l}\right) .
$$

Moreover, write $d_{I_{j}}:=\operatorname{det}\left(X_{\overline{I_{j}}, \bar{J}_{j}}\right)$ for short. Then the lefthand-side of (5) may be written as the $(m-1)$-fold sum

$$
\sum_{\substack{I_{1} \subseteq \mathbf{I}_{0} \\\left|I_{1}\right|=\left|J_{1}\right|}} \operatorname{sgn}\left(\tau_{1}\right) d_{I_{1}} \sum_{\substack{I_{2} \subseteq \mathbf{I}_{1} \\\left|I_{2}\right|=\left|J_{2}\right|}} \operatorname{sgn}\left(\tau_{2}\right) d_{I_{2}} \cdots \sum_{\substack{I_{m-1} \subseteq \mathbf{I}_{m-2} \\\left|I_{m-1}\right|=\left|J_{m-1}\right|}} \operatorname{sgn}\left(\tau_{m-1}\right) d_{I_{m-1}} \operatorname{sgn}\left(\tau_{m}\right) d_{I_{m}} \cdot \operatorname{sgn}(\sigma),
$$

where $I_{m}=\mathbf{I}_{m-2} \backslash I_{m-1}$ and $\sigma=\sigma\left(\mathbf{I}_{[J]},[\tau]\right)$.

Assume $\mathbf{J}_{m-2}=\left\{j_{1} \leqslant \ldots \leqslant j_{a}\right\}, \mathbf{I}_{m-2}=\left\{i_{1} \leqslant \ldots \leqslant i_{a}\right\}$ and $J_{m}=\left\{j_{s_{1}} \leqslant \cdots \leqslant j_{s_{b}}\right\}$. Then the special choice $I_{m}^{\prime}=\left\{i_{s_{1}} \leqslant \cdots \leqslant i_{s_{b}}\right\}$ (i.e., with respect to the relative ordering, " $I_{m}^{\prime}$ is the same subset as $J_{m}$ ") and $I_{m-1}^{\prime}=\mathbf{I}_{m-2} \backslash I_{m}^{\prime}$ determines uniquely a partial permutation $\tau_{m-1}^{\prime}$

$$
\tau_{m-1}^{\prime}: \mathbf{I}_{m-2} \rightarrow \mathbf{J}_{m-2}
$$

According to (4), by construction we have

$$
\operatorname{sgn}\left(\tau_{m-1}^{\prime}\right)=\operatorname{sgn}\left(\tau_{m-1}\right) \cdot \operatorname{sgn}\left(\tau_{m}\right) .
$$

Now consider $\sigma=\sigma\left(\mathbf{I}_{[J]},[\tau]\right)$ in the innermost sum of (6): Erasing the edges corresponding to $\tau_{m-1}$ and $\tau_{m-2}$ from $m_{\sigma}$ and replacing them by the edges corresponding to $\tau_{m-1}^{\prime}$ yields a permutation $\sigma^{\prime}=\tau_{1} \star \cdots \tau_{m-2} \star \tau_{m-1}^{\prime}$ (which, of course, complies to the partition scheme $\left.\left[\tau^{\prime}\right]=\left[\tau_{1} \star \cdots \tau_{m-2} \star \tau_{m-1}^{\prime}\right]\right)$. Since by (4) together with (7) we have

$$
\operatorname{sgn}\left(\tau_{m-1}^{\prime}\right)=\operatorname{sgn}\left(\tau_{m-1} \star \tau_{m}\right) \cdot \operatorname{sgn}\left(I_{m} \unlhd \mathbf{I}_{m-2}\right) \cdot \operatorname{sgn}\left(J_{m} \unlhd \mathbf{J}_{m-2}\right)
$$

and (clearly)

$$
\sigma \backslash\left(\tau_{m-1} \star \tau_{m}\right)=\sigma^{\prime} \backslash \tau_{m-1}^{\prime},
$$

we also have (again by (4))

$$
\operatorname{sgn}(\sigma)=\operatorname{sgn}\left(I_{m} \unlhd \mathbf{I}_{m-2}\right) \cdot \operatorname{sgn}\left(J_{m} \unlhd \mathbf{J}_{m-2}\right) \cdot \operatorname{sgn}\left(\sigma^{\prime}\right) .
$$


Hence the innermost sum of (6) can be written as

$$
\operatorname{sgn}\left(\tau_{m-1}^{\prime}\right) \cdot\left(\sum_{\substack{I_{m-1} \subseteq \mathbf{I}_{m-2} \\\left|I_{m-1}\right|=\left|J_{m-1}\right|}} \operatorname{sgn}\left(I_{m} \unlhd \mathbf{I}_{m-2}\right) \cdot \operatorname{sgn}\left(J_{m} \unlhd \mathbf{J}_{m-2}\right) \cdot d_{I_{m-1}} \cdot d_{I_{m}}\right) \cdot \operatorname{sgn}\left(\sigma^{\prime}\right) .
$$

If we can show that this last sum equals $\operatorname{det}(X) \cdot \operatorname{det}\left(X_{\overline{\mathbf{I}_{m-2}}, \overline{\mathbf{J}_{m-2}}}\right)$, then (5) follows by induction, since the $(m-1)$-fold sum in (6) thus reduces to an $(m-2)$-fold sum, which corresponds to the partition-scheme $\left[\tau^{\prime}\right]=\left[\tau_{1} \star \tau_{2} \star \ldots, \tau_{m-2} \star \tau_{m-1}^{\prime}\right]$.

\section{8 (A generalization of) Laplace's theorem}

Luckily, a generalization (see [5, section 148]) of Laplace's Theorem serves as the closer for our argumentation:

Theorem 2. Let $X$ be an $(m+k) \times(m+k)$-matrix, and let $1 \leqslant i_{1}<i_{2}<\cdots<i_{m} \leqslant$ $m+k$ and $1 \leqslant j_{1}<j_{2}<\cdots<j_{m} \leqslant m+k$ be (the indices of) $k$ fixed rows and $k$ fixed columns of $X$. Denote the set of these (indices of) rows and columns by $R$ and $C$, respectively. Consider some fixed subset $I \subseteq R$. Then we have:

$$
\operatorname{det}(X) \cdot \operatorname{det}\left(X_{\bar{R}, \bar{C}}\right)=\sum_{\substack{J \subseteq C,|J|=|I|}} \operatorname{sgn}(I \unlhd R) \cdot \operatorname{sgn}(J \unlhd C) \cdot \operatorname{det}\left(X_{\overline{R \backslash I}} \overline{C \backslash J}\right) \cdot \operatorname{det}\left(X_{\bar{I}, \bar{J}}\right) .
$$

A combinatorial proof for this identity (using an interpretation of determinants as non-intersecting lattice paths) is implicit in [3, proof of Theorem 6], but we shall give a combinatorial argument which employs the ideas presented in this paper.

Proof. Denote by lhs (rhs) the set of signed and weighted objects corresponding to the left-hand side (right-hand side) of (8). We will prove (8) by showing

- that there is a weight-preserving and sign-preserving injection $\phi:$ lhs $\rightarrow$ rhs,

- and that there is as weight-preserving but sign-reversing involution $\psi$ on the set rhs $\backslash \phi($ lhs $)$.

Overlays of green/red (partial) matchings: In the same sense as presented in section 2.1, we may view both lhs and rhs as families of pairs of matchings $\left(m_{\pi}, m_{\sigma}\right)$, where we may draw the first matching $m_{\pi}$ (with green edges) upon the second one $m_{\sigma}$ (with red edges), so that the pairs appear as overlays of green and red matchings.

Figure 7 , which serves as running example in our proof, shows such overlay of matchings belonging to lhs for $m=5, k=4, R=\{2,3,6,8,9\}$ and $C=\{1,2,5,6,8\}$ (whence $\bar{R}=[9] \backslash R=\{1,4,5,7\}$ and $\bar{C}=[9] \backslash C=\{3,4,7,9\})$. More precisely, the picture shows 
the permutation diagrams $m_{\pi}$ and $m_{\sigma}$ corresponding to the pair of partial permutations $(\pi, \sigma)$, where (in 2-line notation)

$$
\pi=\left(\begin{array}{l}
142735698 \\
123456789
\end{array}\right) \text { and } \sigma=\left(\begin{array}{l}
3794 \\
1457
\end{array}\right)
$$

The green edges belonging to $m_{\pi}$ are shown as solid lines, and the red edges belonging to $m_{\sigma}$ are shown as dotted lines.

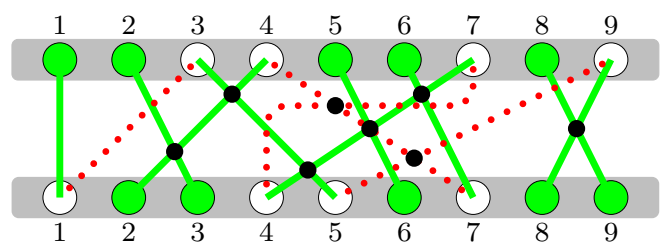

Figure 7: Terms in the expansion of the product of determinants may be viewed as "overlays" of the permutation diagrams of two partial permutations. The red (dotted) edge connecting lower vertex 4 to upper vertex 7 is drawn as curved line for graphical reasons only. The active intersections of edges are indicated by small circles.

This pair $(\pi, \sigma)$ corresponds to the term

$$
(-1)^{6} \cdot\left(x_{1,1} x_{2,4} x_{3,2} x_{4,7} x_{5,3} x_{6,5} x_{7,6} x_{8,9} x_{9,8}\right) \cdot(-1)^{2} \cdot\left(x_{1,3} x_{4,7} x_{5,9} x_{7,4}\right),
$$

which occurs in the expansion of the product of the minors

$$
\operatorname{det}(X) \cdot \operatorname{det}\left(X_{\bar{R}, \bar{C}}\right)
$$

for $X=\left(x_{i, j}\right)_{i, j=1}^{9}$. Obviously, the lower vertices correspond to the rows of $X$, while the upper vertices correspond to the columns of $X$ in Figure 7.

Note that for an overlay of matchings, an intersection of two edges does only contribute to the sign if the edges are of the same colour (both red or both green): We call such intersections active; all other intersections (of edges of different colours) are called inactive (recall section 2.1).

Green, red and uncoloured vertices: In every overlay of matchings in lhs $\cup$ rhs, all upper vertices labelled with numbers from the set $\bar{C}$ and all lower vertices labelled with numbers from the set $\bar{R}$ are incident with a red edge and with a green edge: We call these the uncoloured vertices. All the other vertices are incident with precisely one (either green or red) edge: We assign to them the colour of this single incident edge and call them the coloured (i.e., either green or red) vertices. (All coloured vertices are green in Figure 7.)

\section{Bicoloured paths:}

Obviously, an overlay of matchings constitutes a bipartite graph (with double edges allowed). The connected components of this bipartite graph are either double edges (one green and one red, see the edges connecting lower vertex 4 to upper vertex 7 in Figure 7) or paths 
- whose endpoints are coloured points,

- and whose edges alternate in colour.

We call these components bicoloured paths: Figure 8 shows the two bicoloured paths connecting the lower vertices labelled 3 and 8 with the upper vertices labelled 2 and 1 , respectively, in our running example.

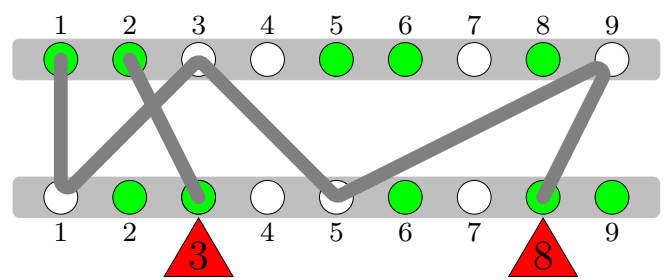

Figure 8: Bicoloured paths (drawn here as thick gray lines) starting in lower vertices 3 and 8 (see the running example in Figure 7).

Obviously, if a bicoloured path connects vertices $x$ and $y$, then

- $x$ and $y$ are on different levels (i.e., one lower and one upper vertex) if and only if $x$ and $y$ have the same colour,

- $x$ and $y$ have different colours (i.e., one green and one red vertex) if and only if $x$ and $y$ are on the same level.

Swapping of colours in bicoloured paths: Observe that for any overlay of matchings, the swapping of the colours (red and green) for all edges in some bicoloured path $p$ simply yields another overlay of matchings with the same absolute weight (since only the colour of edges and vertices does change) and with the same set of uncoloured vertices: We call this operation the swapping of colours in the bicoloured path $p$. (Figure 9 shows the effect of swapping colours in both bicoloured paths from Figure 8: Observe that now there are also red vertices.)

Note that a bicoloured path $p$ might have "inner intersections" (i.e., $p$ may contain intersecting edges), but the swapping of colours in $p$ does not change the status (active or inactive) of such "inner intersections". On the other hand, for every "outer intersection" (of some edge $e_{1}$ belonging to $p$ with some edge $e_{2}$ belonging to another bicoloured path), the status is changed (from active to inactive and vice versa) by swapping colours. So swapping colours in $p$ effects a sign change $(-1)^{k}$, where $k$ is the number of intersections of (edges of) $p$ with (edges of) other bicoloured paths.

The injection $\phi$ : For $\left(m_{\pi}, m_{\sigma}\right) \in$ lhs, we define $\phi\left(m_{\pi}, m_{\sigma}\right)$ by swapping colours in all (distinct!) bicoloured paths starting in the lower vertices labelled by the numbers from $I \subseteq R$. It is easy to see that $\phi$ is an injective mapping lhs $\rightarrow \mathbf{r h s}$ (in fact, it is an involution lhs $\rightarrow \phi$ (lhs)) which preserves (absolute) weights.

In our running example, choose $I=\{3,8\}$ : Figure 9 shows the result of swapping colours in the bicoloured paths shown in Figure 8. The lower vertices with labels in $I$ 
are now red, and the subset of labels of upper red vertices is $J=\{1,2\}$. The overlay of matchings $\left(m_{\pi^{\prime}}, m_{\sigma^{\prime}}\right)$ corresponds to the partial permutations

$$
\pi^{\prime}=\left(\begin{array}{l}
3479568 \\
1245679
\end{array}\right) \text { and } \sigma^{\prime}=\left(\begin{array}{l}
127349 \\
134578
\end{array}\right)
$$

which correspond to the term

$$
(-1)^{5} \cdot\left(x_{1,3} x_{2,4} x_{4,7} x_{5,9} x_{6,5} x_{7,6} x_{9,8}\right) \cdot(-1)^{2} \cdot\left(x_{1,1} x_{3,2} x_{4,7} x_{5,3} x_{7,4} x_{8,9}\right)
$$

occuring in the expansion of the product of the minors

$$
\operatorname{det}\left(X_{\overline{R \backslash I}, \overline{C \backslash J}}\right) \cdot \operatorname{det}\left(X_{\bar{I}, \bar{J}}\right) .
$$

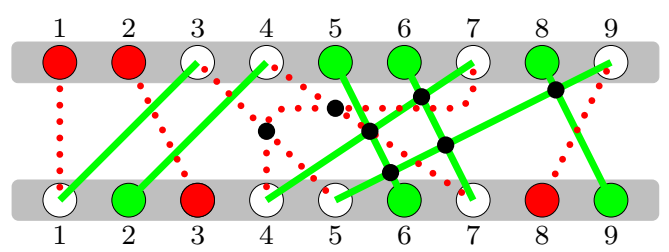

Figure 9: Overlay of matchings obtained by swapping colours in the bicoloured paths (see Figure 8) starting in lower vertices 3 and 8 (see the running example in Figure 7).

The mapping $\phi$ is not injective, so we really need the involution $\psi$ : So far we found an injection $\phi:$ lhs $\rightarrow$ rhs which preserves absolute weights: We need to show yet

- that the change of sign effected by $\phi$ equals $\operatorname{sgn}(I \unlhd R) \cdot \operatorname{sgn}(J \unlhd C)$,

- and that the total weight of rhs $\backslash \phi$ (lhs) equals 0 .

Figure 10 demonstrates that (in general) $\phi$ is not surjective (whence $\mathbf{r h s} \backslash \phi(\mathbf{l h s}) \neq \emptyset$ ): The overlay of matchings $\left(m_{\pi^{\prime \prime}}, m_{\sigma^{\prime \prime}}\right)$ depicted there corresponds to the partial permutations

$$
\pi^{\prime \prime}=\left(\begin{array}{l}
5346789 \\
1245679
\end{array}\right) \text { and } \sigma^{\prime \prime}=\left(\begin{array}{l}
123479 \\
134578
\end{array}\right)
$$

which correspond to the term

$$
(-1)^{2} \cdot\left(x_{1,5} x_{2,3} x_{4,4} x_{5,6} x_{6,7} x_{7,8} x_{9,9}\right) \cdot(-1)^{0} \cdot\left(x_{1,1} x_{3,2} x_{4,3} x_{5,4} x_{7,7} x_{8,9}\right)
$$

also occuring in the expansion of the product of the minors

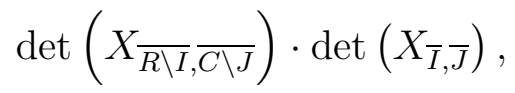


but not occurring as $\phi(z)$ for any $z \in$ lhs, since there is a bicoloured path starting in the upper vertex with label 1 , which also ends in an upper vertex (with label 5): This cannot happen in overlays belonging to lhs. It is easy to see that every element of rhs $\backslash \phi(\mathbf{l h s})$ contains a bicoloured path starting and ending in upper vertices, so the involution $\psi$ suggests itself: Identify the leftmost upper vertex which is connected to another upper vertex by a bicoloured path $p$, and swap colours in $p$. This clearly defines an involution preserving absolute weights: It remains to show that $\psi$ is sign-reversing, so that the total weight of rhs $\backslash \phi$ (lhs) equals 0 .

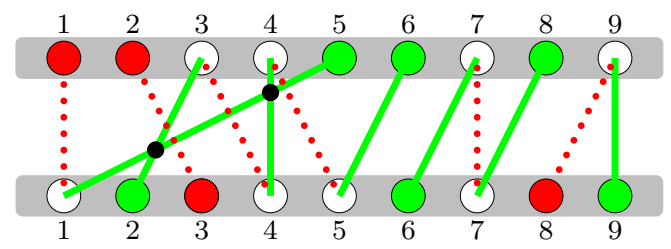

Figure 10: An overlay of two matchings belonging to rhs which does not belong to $\phi$ (lhs).

Sign changes effected by swapping colours in bicoloured paths: Bicoloured paths are connections of coloured points, which we may simply indicate by corresponding edges (see Figures 11 and 12). Observe that two such edges corresponding to (different) bicoloured paths $p_{1}$ and $p_{2}$ have an intersection if and only if $p_{1}$ and $p_{2}$ have an odd number of intersections, and recall that swapping the colours in some bicoloured path $p$ yields a sign change of $(-1)^{k}$, where $k$ is the number of "outer intersections" of $p$ (i.e., intersections with other bicoloured paths).

If we forget the uncoloured points in Figure 11, we recognize a permutation diagram: It is clear that for every overlay of matchings from lhs, we obtain such permutation diagram.

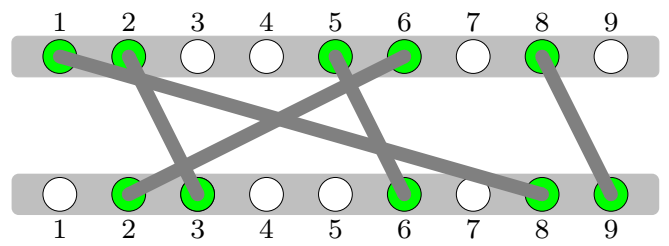

Figure 11: The edges corresponding to the connections by bicoloured paths in Figure 7 .

Denote by $B$ the set of all bicoloured paths in an overlay of matchings from lhs and consider some subset $S \subset B$ and its complement $\bar{S}=B \backslash S$. By swapping colours of all bicoloured paths in $S$, the status (active/inactive) of every "inner intersection" of $S$ (i.e., an intersection of some $p \in S$ with another $p^{\prime} \in S$, where $p=p^{\prime}$ is possible) is unchanged, while the status of every "outer intersection" of $S$ (i.e., an intersection of some path $p \in S$ 
with some path $q \in \bar{S}$ ) is swapped (from active to inactive and vice versa): So the sign change equals $(-1)^{k}$, where $k$ is the number of "outer intersections" of $S$. But this is the same sign change we encounter if we partition the permutation corresponding to $B$ into the two partial permutations corresponding to $S$ and $\bar{S}$, respectively, in the sense of Lemma 1: So by (4), this sign equals $\operatorname{sgn}(I \unlhd R) \cdot \operatorname{sgn}(J \unlhd C)$, and the injection $\phi$ is sign-preserving.

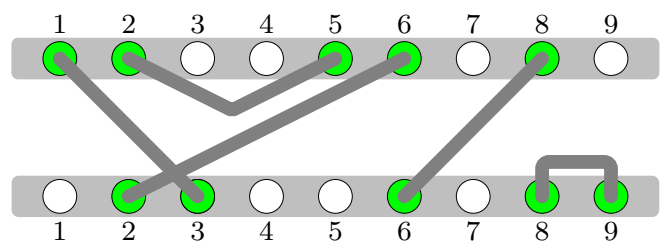

Figure 12: The edges corresponding to the connections by bicoloured paths in Figure 10 .

By the same reasoning, we see that the sign change effected by swapping some bicoloured path connecting two upper vertices $x$ and $y$ (see Figure 13) is $(-1)^{k}$, where $k$ is the number of coloured vertices lying between $x$ and $y$. Assuming that $x$ is the $i$-th and $y$ is the $(i+k+1)$-the element of the ordered set $C$ (which is the set of upper coloured vertices), such swapping replaces factor $(-1)^{i}$ by $(-1)^{i+k+1}$ (or vice versa) in $\operatorname{sgn}(J \unlhd C)$, which gives sign change $(-1)^{k+1}$ : Altogether, such swapping yields sign change $(-1)^{2 k+1}=-1$, whence the involution $\psi$ is sign-reversing.

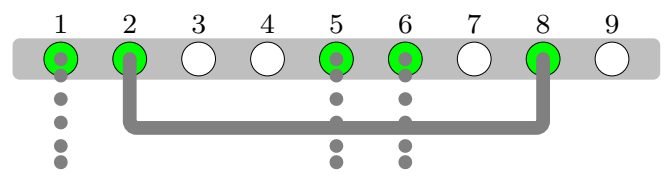

Figure 13: An edge corresponding to a connection between two upper vertices.

\section{Acknowledgement}

I thank the anonymous referee for a very careful reading of the manuscript and helpful comments and suggestions.

\section{References}

[1] A.C. Aitken. Determinants and Matrices. Oliver \& Boyd, Ltd., Edinburgh, 9th. edition, 1956. 
[2] S. Caracciolo, A.D. Sokal, and A. Sportiello. Algebraic/combinatorial proofs of Cayleytype identities for derivatives of determinants and pfaffians. Advances in Applied Mathematics, 50(4):474 - 594, 2013.

[3] M. Fulmek. Viewing determinants as nonintersecting lattice paths yields classical determinantal identities bijectively. Electron. J. Combin., 19(3):\#P21, 2012.

[4] T. Muir. The Theory of Determinants in the historical order of development. MacMillan and Co., Limited, London, 1906-1923.

[5] T. Muir. A Treatise on the Theory of Determinants. Longmans, Green and Co., London, 1933.

[6] G. Vivanti. Alcune formole relative all'operazione $\Omega$. Rend. Circ. Mat. Palermo, 1890. 